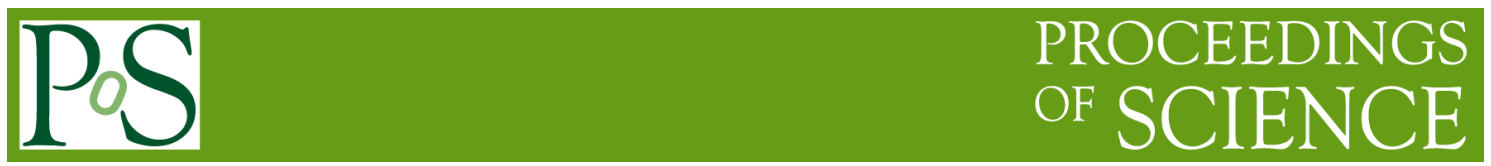

\title{
American Particle and Nuclear Physics Planning
}

\section{Hugh E. Montgomery ${ }^{1}$}

Jefferson Lab

12000 Jefferson Avenue, Newport News, VA 23693, USA

E-mail: montejlab.org

In the United States the planning process relevant to future deep inelastic scattering involves both the high energy physics and nuclear physics funding and the two communities. In Canada there is no such split between the communities. Within the past two years there have been several planning initiatives and there may be more to come. We review the current status of both the planning and the plans.

XXII. International Workshop on Deep-Inelastic Scattering and Related Subjects 28 April - 2 May 2014

Warsaw, Poland

1

Speaker 


\section{Introduction}

In the United States, the dominant source of funding for facilities for the physical sciences is the Department of Energy Office of Science (SC) although the National Science Foundation (NSF) is also a strong player. The Office of Science is divided into several Associate Directorates, of which the Office of High Energy Physics (OHEP) and the Office of Nuclear Physics (ONP) fund facilities of interest to this conference. In partnership with the NSF, the Office of Science charges advisory committees, the High Energy Advisory Panel (HEPAP) and the Nuclear Science Advisory Committee (NSAC), to provide congressionally mandated advice on directions of the programs. These committees and their subcommittees draw their membership from the physicists in the field including some representation from the international communities. In this paper, I will concentrate on accelerator-based facilities and will include TRI-Universities Meson Factory (TRIUMF), the Canadian accelerator laboratory, which received approval for its next five year plan in a sequence of reviews started in 2013 and completed earlier this year.

\section{Particle Physics}

In particle physics, the global program, as seen from the United States, has changed dramatically with the closure of the Tevatron at Fermilab and PEPII at SLAC. These major components have been in many senses replaced by the Large Hadron Collider (LHC), and the two general purpose detectors, ATLAS and CMS. In fact the LHC Project was the largest US particle physics project inside the past twenty years. At one point it appeared as though the International Linear Collider was a possible thrust for the next large US construction but circumstances mitigated against that also. So to first approximation, the highest energies (The Energy Frontier) are no longer on this continent. The primary vector at SLAC has developed a large cosmic physics (Cosmic Frontier) component with the FERMI satellite gamma ray experiment. That component has also been present at Fermilab with the Dark Energy Survey and the support of a suite of Dark Matter experiments.

Beyond the Energy Frontier, and the Cosmic Frontier, there is the remaining segment of accelerator-based particle physics dubbed the Intensity Frontier. The emphasis on intensity reflects the rarity of the events which are sought in this arena. Neutrino cross-sections are small and the oscillation phenomena require large numbers of events to study the characteristics and to determine the parameters. Similarly searches for phenomena and events never seen before require large numbers at the source, for example the search for the conversion of muons to electrons.

Since the early eighties, the particle physics community has periodically retreated en masse to Snowmass in the Colorado Rockies. Needing a similar gathering in 2013, it chose to conduct a serious of preparatory workshops to prepare for a large community workshop[1] in Minneapolis. This large study provided voluminous input to the deliberations of the Particle Physics Project Prioritization Panel (P5), a sub-panel of HEPAP, which took up its charge in the Fall of 2013. Much 
of the material in the sections on particle physics, which follow, contain material prepared as input to the $\mathrm{P} 5$ process[2].

\subsection{Energy Frontier}

Participation in the LHC program by physicists from US institutions is at a level at which they represent the largest national group in each of the large experiments, about $25 \%$ in ATLAS and nearer $30 \%$ in CMS. The case for the strong participation is that this is the only place in the world which currently provides access to the exciting physics associated with the Higgs mechanism and perhaps the new physics beyond what we know as the standard model. The two experimental groups made a joint presentation, the experiments need their strength, they need the experiments and the contributions to the machine are also mutually supported. The question for P5 is presumably not "YES" or "NO", rather at what level of support.

The discovery of the Higgs particle was the event foreseen by the participants in the design and study of the International Linear Collider, the superconducting radio-frequency linear collider has a design energy of $500 \mathrm{GeV}$. In the past year, there has been strong interest and lots of political action in Japan. For example, a site has been selected and it seems that Japanese delegates have made approaches to the US and others. It is my view that physics is best served by a global balance and, following the European study publication of a year ago, encouragement in some useful form of the International Linear Collider hosted by Japan has many attractions. This is a specially interesting challenge for P5.

\subsection{Intensity Frontier - Neutrino Physics}

Over the past twenty years, Fermilab has systematically expanded its stake in neutrino physics. Since the dawn of the Tevatron it has been a significant player in high energy neutrino physics. With the experiment sending beam to the MINOS experiment in a mine in Minnesota $750 \mathrm{~km}$ away it entered the long baseline oscillation physics arena. With MiniBooNE it sought confirmation of the Los Alamos results. Along with corresponding "near" detectors there are half a dozen individual neutrino detectors in operation on-site at Fermilab. The MINOS detector, thought to be large at the time of its debut, is now dwarfed by the NOvA[3] detector, see Figure 1, which is off-axis with respect to the beam and is even further north, but still just inside the United States. That detector contains about 14 kilotons of plastic and liquid scintillator chosen to be sensitive to electrons and so to continue the exploration of the oscillation of muon neutrinos into electron neutrinos. The proton accelerator complex at Fermilab is set to provide $700 \mathrm{~kW}$ of beam to the current suite of experiments. The NOvA experiment has detected its first neutrinos and will be operational this year.

The new long baseline initiative (LBNE)[4] is even more ambitious. A new beamline from Fermilab to South Dakota would provide a baseline of $1300 \mathrm{~km}$; see Figure 2 . The chosen detector technology is a liquid Argon detector which is judged to be higher performance than a water Cerenkov detector such as has been used at Kamiokande in Japan. Ideally, the detector would be 
placed underground providing an environment which would permit the use of the detector to search for the kaon mode of proton decay. For all the possible physics goals of the detector, a large mass is a prerequisite. But of course, depth, and size drive the cost. And, whatever the choice of detector construction, the beamline at Fermilab will consume considerable resources as will the enhancement of the proton source accelerator complex. Again, higher power is the name of the game, and 1.2 MW is very desirable. Altogether, this is a large project and understanding what to do with the LBNE initiative is probably one of the more difficult components of the P5 work.

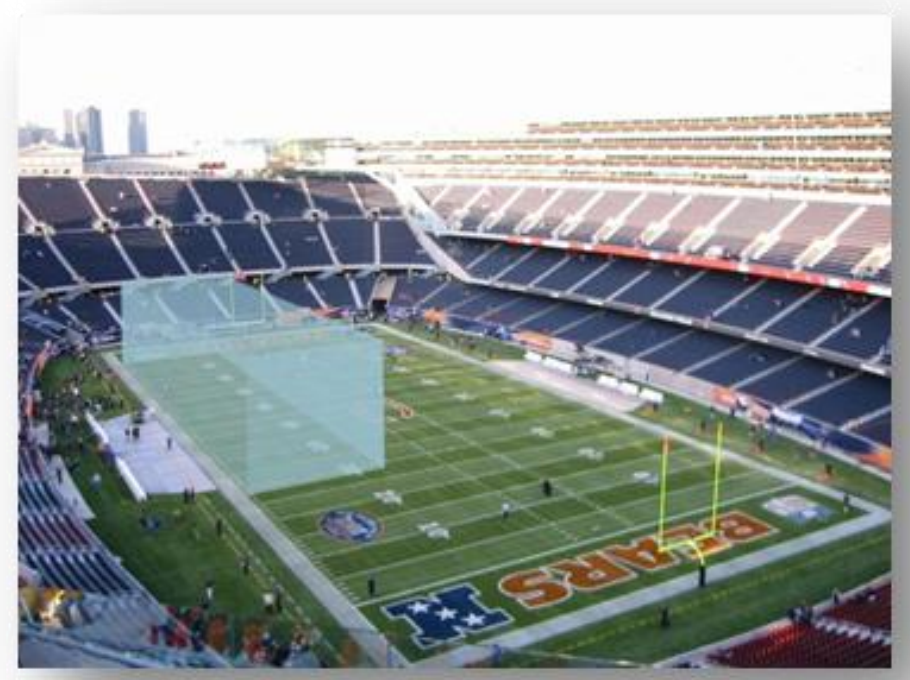

Figure 1. Illustration of the NOvA detector set inside a football stadium to set the scale.

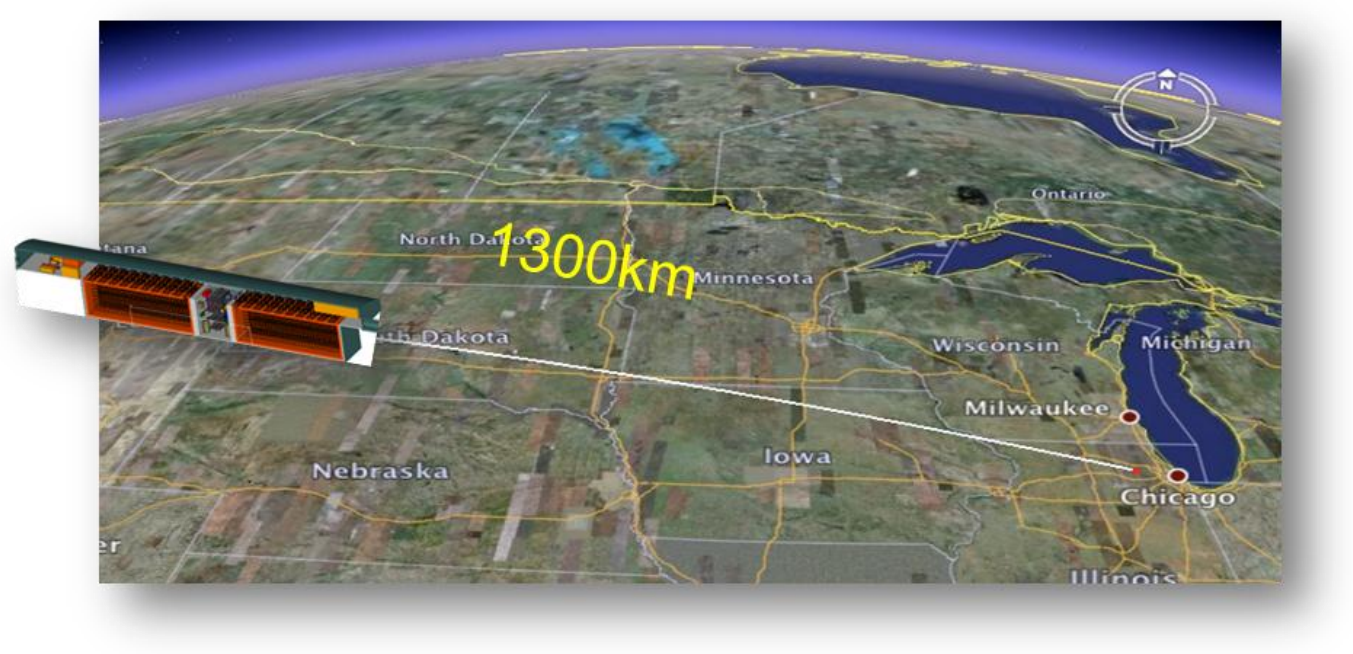

Figure 2. A high altitude view of the LBNE trajectory from Fermilab to South Dakota. 


\subsection{Intensity Frontier - Muon Physics}

In principle the definition of the scope of the Intensity Frontier includes all varieties of flavour physics, beauty and kaons. Recently at Fermilab, emphasis has been given to muon based physics. The proton source is able to provide intense muon beams which allow an enhancement of the search for flavour violating conversions of muons to electrons. The current limits are at the $10^{-13}$ level and the goal of the $\mu 2 \mathrm{e}[5]$ experiment is a single event sensitivity (SES) of about $10^{-17}$. There is competition from the COMET experiment at JPARC in Japan, which has a similar goal on a similar schedule.

The measurement of the muon anomalous magnetic moment $(\mathrm{g}-2)_{\mu}$ has been of interest for the past several decades with experiment challenging theory and vice versa. After the recent round of jousting, the theory and experiment indicated a small difference, enough to be intriguing, not enough to be definitive. So the solution was, of course, to improve the experiment. The large magnet was transported from Brookhaven National Lab to Fermilab and is being installed. The goals of the experiment[6] are a factor of five or so reduction in the experimental uncertainty. If the central values of experiment and theory remained as they are now, a disagreement in excess of seven standard deviations would presumably be convincing.

My own judgment is that, while far from negligible, these two projects are sufficiently well advanced and established that P5 will provide unequivocal support.

It is also interesting to note that searches for new phenomena are now being conducted on relatively low energy machines; for example, CEBAF at Jefferson Lab where there are three active searches for the A' boson a popular candidate as a source of dark matter.

\subsection{Cosmic Frontier}

Within the United States, the interests of those considered to be particle physicists have always been broad and over the past thirty years increasing resources have shifted towards the searches for dark matter, and more recently, dark energy; broadly the origin and evolution of the universe. Great value accrued from exploiting some of the detector and computational techniques of particle physics. Conversely of course, the resources devoted to this segment of particle physics have increased to become a substantial fraction of the high energy physics budget. I excuse myself from spending proportional time on this by noting that the same partition of physics does not occur in all parts of the world, and that the overlap with Deep Inelastic Scattering, except through the resource limitations, is small.

\subsection{The P5 Process}

In his report to HEPAP in March, the Chair of P5, Steve Ritz, laid out the physics drivers as seen by P5 leading with the Higgs, and the physics around it, neutrinos and their mass characteristics, dark matter and dark energy, the search for new phenomena, particles and interactions. 
In talking about optimizing the program, Ritz looked to balance the science driven picture with the need to have a prioritized portfolio for discovery. That portfolio needs to be informed by the international context. This can imply that the physicist should follow the opportunity wherever in the world it presents itself, but recognising that reliable partnerships and sensitivity to duplications and complementarity are very important. The international aspect was a strong thread.

Of course when the crunch comes, the question is how to choose specific projects to be supported. The questions can start out being similar, for example the physics potential has to be there. One should also ask what the potential is for the project to alter the thrust of the field. One question the proponent does not like has to do with the issue of the immediate needs of the field. It may be that waiting may clarify some aspect of the physics and obviate the need for a project. So a measured approach can also permit the use of time as a variable to mitigate conflict. Of course serious emphasis must be given to the estimation of required resources, and in the end there is a responsibility to attempt to maintain a strong participation in the global field, which should mean a leadership position for the US in some parts, not all, of the field.

\section{Canada}

The larger laboratories in Canada include the Perimeter Institute, SnoLab and TRIUMF. The latter serves as the lead laboratory for most of Canadian particle and nuclear physics. In the latter part of 2013, the National Research Council, which provides the base funding for TRIUMF convened a series of reviews to consider the plan[7] advanced by the laboratory for the next five years. The program was very strong both in its international participation and contributions and in its local program.

Through the expertise at the laboratory Canada has made major contributions to international facilities such as HERA, T2K at JPARC in Japan, and to the Large Hadron Collider program. The intent is to maintain a strong presence in the latter two initiatives. Canada has had quite a strong leadership role in the T2K experiment as well as contributing to the JPARC machine. Likewise there are contributions to the LHC machine and to the ATLAS experiment and it is felt imperative to maintain or enhance the strength of that program.

What is also impressive is the local accelerator complex. The ISAC facility is a high power $(50 \mathrm{~kW})$ ISOL facility for rare isotope beams. Its techniques are complementary to others in the US and rather nicely will complete the picture for North America. There is also an aggressive program to construct a superconducting radiofrequency electron linac to enhance the ISAC facility.

Overall the lab is a significant contributor to North American and inter-regional sub-atomic physics.

\section{Nuclear Physics}

The most recent Long Range Plan[8] for nuclear physics was completed in 2007. In that report, the first four recommendations emphasized the completion of the $12 \mathrm{GeV}$ Continuous 
Electron Beam Accelerator Facility (CEBAF) Upgrade Project[9] at Jefferson Lab, the start of construction of the Facility for Rare Isotope Beams (FRIB), a targeted program to address the physics of neutrinos and fundamental symmetries, and fourth the luminosity upgrade of the Relativistic Heavy Ion Collider (RHIC)[10] and of its experiments. An unnumbered recommendation also encouraged the development of an Electron Ion Collider. At the present time, the $12 \mathrm{GeV}$ CEBAF Upgrade project is well advanced with accelerator commissioning successfully started. The FRIB[11] facility, as of earlier this year, is under construction at the National Superconducting Cyclotron Laboratory (NSCL) at Michigan State University (MSU); it is a multihundred million dollar initiative. The other two recommendations, concerning fundamental symmetries and RHIC, have also seen progress; the luminosity upgrade of RHIC is complete. This spells remarkable success, although the constraints associated with executing this program led to an NSAC subcommittee being formed in 2012-2013 to advise on implementation of the plan. The recommendations did differentiate between the three major components of the program assuming a flat base budget but argued for modest growth, which was then supported by the President's budget request. It should be noted that the Holifield Radioactive Ion Beam Facility (HRIBF) at Oak Ridge was closed in 2012.

In spring of this year, a charge has been given to NSAC to develop a new Long Range Plan. Since the field has conducted this exercise on several occasions starting in the early nineteen eighties, the process is well understood. The engagement of the American Physical Society Division of Nuclear Physics which organizes Town Meetings and the production of white papers, is in progress. Chairmanship, membership of the committees and selection of convenors for the process are under consideration. The report is requested by October 2015.

\subsection{Low Energy Nuclear Structure}

The existing large nuclear structure facilities in the US are the ATLAS (Argonne Tandem Linac Accelerator System) at Argonne National Lab and the NSCL facility at MSU which are complemented by smaller facilities at Texas A\&M U., Lawrence Berkeley Lab, U. Washington, and U. Kentucky supported by DOE and Florida State U. and Notre Dame U. supported by NSF. The two large facilities support about three hundred users each and provide complementary opportunities. While NSCL concentrates on unstable isotope beams, ATLAS provides a full range of stable isotope beams and has recently brought online a facility to provide rare isotopes resulting from the fission of ${ }^{252}$ Californium.

Although preparation for the major new facility FRIB at NSCL has been underway for some time, it was only with the 2014 appropriation that the project could formally start construction. The project represents a major investment, which is primarily the responsibility of DOE, however a very substantial contribution is provided by the State of Michigan through MSU.

The physics goals put a premium on exploration of a large number of isotopes, and hence on the time taken to make measurements with each. In turn this is controlled by the supportable beam power. The facility will provide $400 \mathrm{~kW}$ beam power $\left(5.10^{13}{ }^{238} \mathrm{U}^{-1} \mathrm{~s}^{-1}\right)$. The primary production 
process for the radioactive beams involves separation of the isotopes in flight. The project is expected to be complete around the end of the decade.

\subsection{Relativistic Heavy Ion Physics}

The heavy ion physics program has several components with participation in the LHC experiments as well as RHIC experiments at Brookhaven National Lab. Thus far the program at RHIC features a broader range of heavy ion species and a flexibility in choice of energy which is not (yet?) available on the LHC. The luminosity upgrades of RHIC using three dimensional stochastic cooling have been completed and a new source is in use. Silicon vertex detectors have been introduced to both of the experiments PHENIX and STAR. There are other ideas for enhancement of machine and experiments being considered.

There has been considerable emphasis in the past couple of years on the spin physics program using proton beams. This is an opportunity to try to tie down the contribution of the gluon field to the spin of the nucleon. At the time of writing there are hints of a finite gluon contribution but the results are not yet definitive.

With the new vertex detectors, the experiments are now better equipped to explore the heavy flavor aspects of the quark-gluon plasma. Understanding how the heavy flavor states propagate can clarify the details of the mechanisms in play. A program over the next three years of operation is foreseen. Using the flexibility in energy, attempts have been made to map out the behavior of the quark-gluon plasma in order to identify the boundaries of the apparent phase transition and to seek a critical point. Thus far the results are not conclusive and a plan is evolving to mount a strong campaign in the period 2018-2019 to dramatically improve the statistics at the lower energies, especially below $20 \mathrm{GeV}$. Beyond 2019, the program depends on what improvements to the complex, accelerator and detectors might be approved. However, it is imagined that a more incisive study of the jet characteristics and further studies of quarkonium would be fruitful.

\subsection{Medium Energy Physics}

The RHIC spin program along with the SeaQuest Drell-Yan experiment at Fermilab both contribute significantly to the understanding of the nucleon and the nucleus. Nevertheless, the dominant component of the Medium Energy Physics program is conducted with CEBAF at Jefferson Lab. Experiments with beams up to $6 \mathrm{GeV}$ were conducted up to two years ago. An eighteen month shutdown permitted installation of ten new superconducting cryo-modules and the refurbishment of the magnet arcs. Commissioning of the new machine over the past several months has demonstrated the capability to support $12 \mathrm{GeV}$ and 5.5 pass beam with $10.5 \mathrm{GeV}$ has been achieved. The new experimental layout will include a fourth hall with a new photon-based spectroscopy experiment, GlueX. The equipment in the existing three halls is also undergoing substantial upgrade. NSF and international support for the detector work has been very important.

The goal is to support of order 30 weeks of operation each year as the transition from commissioning to physics is made in the different Halls. The GlueX experiment will search for 
hybrid and exotic meson states. The kinematics of the machine, and its ability to support very high luminosity experiments, with very high electron beam and target polarizations, make the multidimensional exploration of the valence characteristics of the quarks and gluons accessible. These special features of the machine broaden the scope of the program to include in-depth understanding of hadronization of partons. Elsewhere we mention the potential of parity violation experiments to explore fundamental symmetries and there are also three proposals approved to search for new bosons analogous to the photon but with some modest mass. The access to the weak interaction permits the investigation of the distribution of neutrons in nuclei and the determination of the "neutron radius". This can connect to several aspects of nuclear structure but also to the understanding of neutron stars. Overall, there are experiments in the pipeline which constitute a very strong physics program for almost a decade.

\subsection{Fundamental Symmetries}

The demarcation between particle and nuclear physics funding involves a complex set of considerations such as the origin of the experimental techniques, the evolving interests of renowned principal investigators, and the physics goals themselves. Thus in the nuclear physics portfolio, we find neutrino-less double beta decay experiments to try and identify the character of neutrino mass. Is it Dirac, or Majorana? The determination of the absolute mass of the neutrinos by studying the end point of beta decay spectra is also included, as are the searches for electric dipole moments of, for example, the neutron. The ability to control the spin of high power electron beams has provided access to important low energy measurements which test the standard model. One example was the E158 Moller scattering experiment at SLAC, but recently a powerful program of parity violation measurements has emerged at Jefferson Lab. Recently measurements have been made of the weak charge of the proton and of parity violation in deep inelastic scattering, advancing the precision by a factor of five was published in Nature. Two major parity violating programs at Jefferson Lab, one a new measurement[12] of Moller scattering, are being advanced. ONP has decided to create a coherent program from the suite of measurements which fall into this Fundamental Symmetry category. The $0 v \beta \beta$ program has been the focus of recent interest and NSAC responded to a charge to look at how that field might evolve. The McKeown sub-committee reported on its initial findings with a report at the most recent NSAC meeting.

\subsection{Electron Ion Collider}

Above, we have discussed the scope of physics which can be attacked by the combination of the RHIC machine and the CEBAF $12 \mathrm{GeV}$ machine. The latter, with its very high luminosity is ideally suited to explore with exquisite precision the valence quark regions of the nucleon whether free or embedded in a nucleus. However, we know that the valence quarks are far from the complete picture. Most of the mass of the world around us comes from the strong interactions, the exchange of gluons. At low fractional momentum of the nucleon, the population of partons is predominantly made up of gluons. To attain this low $\mathrm{x}_{\mathrm{Bj}}$, with a substantial momentum transfer, demands more energy than provided by CEBAF. Already in the 2007 NSAC Long Range Plan the 
idea of an Electron Ion Collider to provide a next generation QCD facility in the United States was discussed.

Over the past few years, led by the Jefferson Lab and RHIC communities, a consensus[13] has emerged that to satisfy the physics needs, such a machine should have high luminosity in the range $10^{33}-10^{34} \mathrm{~cm}^{-2} \cdot \mathrm{s}^{-1}$, high polarization $(\sim 70 \%)$ in both beams, and an energy capability from $20-$ $\sim 100 \mathrm{GeV}$. One notes how RHIC and Jefferson Lab communities started from quite different inherited machine configurations but converged. It is intended that a common physics case be made to the 2015 Long Range Plan process. However, each lab has developed a design for a complete complex aimed at achieving the desired parameters with the thought that a down select be made at the CD1 stage of the project.

It is possible to contemplate a possible timeline for the construction of an electron ion collider. A possible view is illustrated in Figure 4. The constraints are that within the portfolio of ONP, the $12 \mathrm{GeV}$ Upgrade project at Jefferson Lab is still to be completed. The FRIB construction project is now in construction and will complete around the end of the decade. It is not expected that a new construction project could be supported before that time. From the perspective of the approval process, endorsement from a Long Range Plan is a prerequisite, and subsequent critical decision steps from CD0 through CD1, CD2 and CD3 typically consume up to 12-18 months each. The two views are grossly consistent.

There appears to be considerable interest from the international nuclear physics community and it would be enormously helpful if we could incorporate their thinking into the Long Range Plan process.

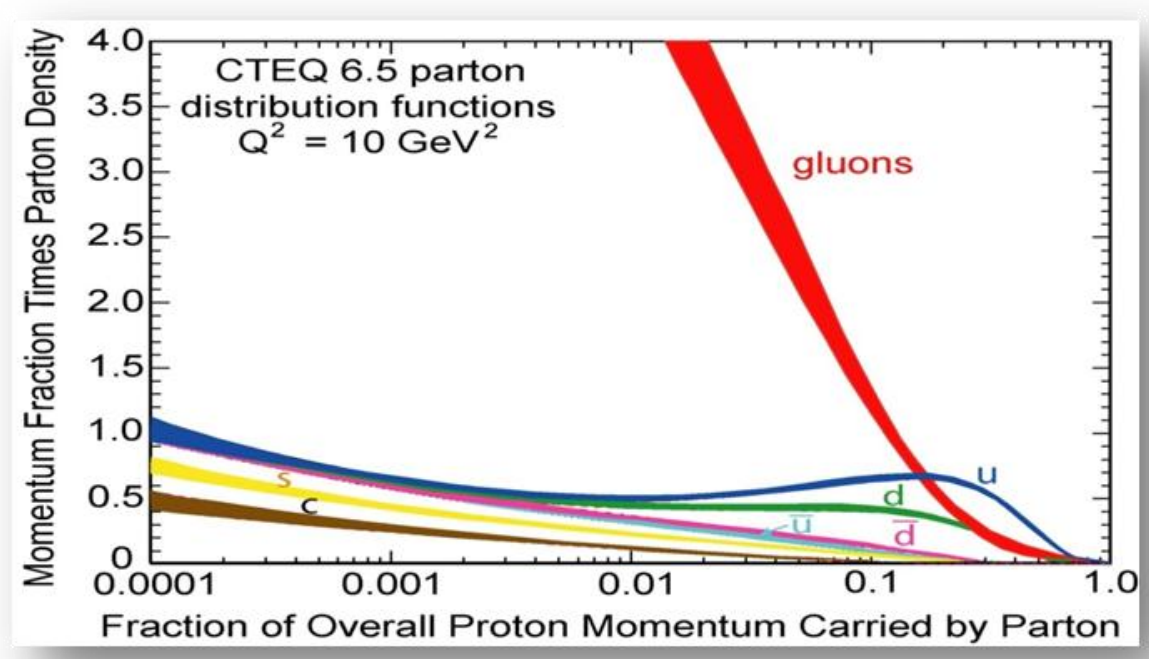

Figure 3. Parton distribution functions, which demonstrate the importance of exploring the region with $0.1>\mathrm{x}_{\mathrm{Bj}}>0.001$. 


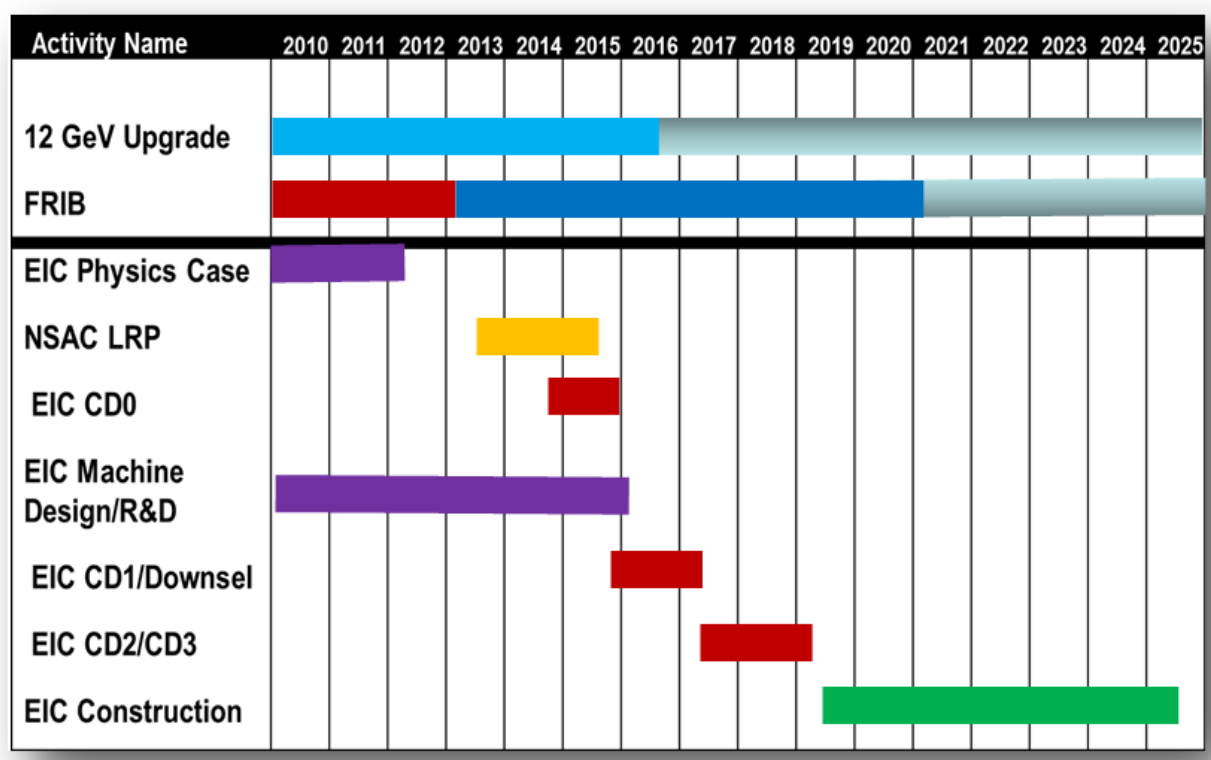

Figure 4. Notional time line for preparation and construction of an electron ion collider. Also shown are approximate construction times for projects currently in construction.

\section{Conclusions}

I hope I have conveyed the sense that the processes used by both particle and nuclear physics to set the course of the research in North America involves both the communities and the DPF and the DNP of the American Physical Society, as well as a formal process based on charges received from the responsible government agencies. The presentation by the Chair of P5 to HEPAP showed a comparison between the requested funding and the guidance provided. In 2012-13 the motivation for the charge to NSAC and the Tribble sub-committee was the budget constraint on the long range plan for nuclear physics laid out in 2007. It is clear that budgetary constraints necessitate difficult choices so the engagement of the communities is enormously important.

At the time of the talk, P5 had not yet reported on its conclusions, but I take the liberty to include the main conclusions here. Support for a continued strong participation in the Large Hadron Collider at CERN, both in the machine and in the experiments is clear and unequivocal, but it is recognized that it cannot be the whole program. There was strong endorsement of the scientific case for the International Linear Collider and some support, although measured, for help in development of the initiative to build the machine in Japan, under all budget scenarios. The muon program at Fermilab, with the measurement of $(\mathrm{g}-2)_{\mu}$ and the search for conversion of muons to electrons, was seen as eminently achievable. The development, in collaboration with international partners of a coherent short - and long-baseline neutrino program, hosted at Fermilab was urged. It was recommended that a new international collaboration of partners be formed to design and build the new long baseline neutrino facility (LBNF). Emphasis was placed on the need for new ideas as well 
as more resources to achieve very important physics goals. Beyond these major recommendations, the report had a lot to say about the suites of smaller projects which would attack a number of issues including dark matter and dark energy.

In nuclear physics the components of the program for the next several years are already in play. RHIC is operational and a component of the heavy ion community works on the Large Hadron Collider. The $12 \mathrm{GeV}$ Upgrade of CEBAF at Jefferson Lab will be the basis for the next decade of Medium Energy physics and the parity violating electron scattering component of the Fundamental Symmetries program. The latter envisages advances in the exploration of $\mathrm{CP}$ violation through exploration of electric dipole moments and progress towards detection of the $0 v \beta \beta$ decay process. The Long Range Plan recently instigated and should report late in 2015. The issues will concern the relative emphases of these components and importantly the prospects of an Electron Ion Collider in America.

In his March presentation to HEPAP, one complete transparency of Ritz' talk was devoted to the emphasis that particle physics is global and it was a prominent theme in the final report. I would also say that a similar argument can be made but a little less stridently that this applies also to nuclear physics. Certainly if one bases the judgment on the fractions of the user communities of the US facilities, which are non-US, it is clear. In either case, it makes absolute sense for the planning processes on the different continents to attempt a high degree of complementarity.

\section{Acknowledgements}

In the preparation of the talk and of this paper, people responded enthusiastically to my requests for help or for the opportunity to discuss the situation as they saw it. In particular, I would like to thank Greg Bock, Rolf Ent, Andy Lankford, Nigel Lockyer, Bob McKeown, Allison Lung, Lia Merminga, Berndt Mueller, Steve Ritz, and Brad Sherill. I have also availed myself of the material on the P5 Web pages. The preparation of the talk and this paper depended heavily on the skills of Mary Beth Stewart. Finally, I would like to thank the organizers of the DIS 2014 workshop; while my attendance was abbreviated, the hospitality was impeccable.

\section{References}

[1] Community Summer Study Snowmass 2013, http://www.snowmass2013.org/tiki-index.php

[2] Particle Physics Project Prioritization Panel (P5), http://usparticlephysics.org/p5

[3] NOvA Neutrino Experiment, http://www-nova.fnal.gov/

[4] Long Baseline Neutrino Experiment (LBNE), http://lbne.fnal.gov/

[5] Mu2e muon to electron conversion experiment, http://mu2e.fnal.gov/

[6] Muon g-2, http://muon-g-2.fnal.gov/ 
[7] T. Meyer Ed., TRIUMF Five-Year Plan 2015-2020, Realising the Vision, http://www.triumf.ca/fiveyear-plan-2015\%E2\%80\%932020

[8] The Frontiers of Nuclear Science, A Long Range Plan, The DOE Nuclear Science Advisory Committee, December 2007, http://science.energy.gov/np/nsac/

[9] $12 \mathrm{GeV}$ Upgrade Project, http://www.jlab.org/12GeV/public.html

[10] Relativistic Heavy Ion Collider, http://www.bnl.gov/rhic/

[11] FRIB, http://www.nscl.msu.edu/features/facility-rare-isotope-beams-frib

[12] The Moller Experiment at Jefferson Lab, http://hallaweb.jlab.org/12GeV/Moller/

[13] A. Accardi et al., Electron Ion Collider: The Next QCD Frontier - Understanding the glue that binds us all, http://arxiv.org/abs/1212.1701 